\title{
SPECTRAL ASYMPTOTICS FOR TOEPLITZ MATRICES GENERATED BY THE POISSON-CHARLIER POLYNOMIALS
}

\author{
GEORGI E. KARADZHOV
}

(Communicated by Palle E. T. Jorgensen)

\begin{abstract}
A conjecture of Grenander and Szegö for the traces of Toeplitz matrices generated by the Poisson-Charlier polynomials is proved.
\end{abstract}

The Poisson-Charlier polynomials $\left\{p_{m}(x)\right\}, m \geq 0$, are defined on the set $X$ of all nonnegative integers $[6, p .34]$. This is a complete system of orthonormal polynomials in the space $l^{2}=l^{2}(X, j(x))$, where $j(x)=e^{-a} a^{x}(x !)^{-1}, a>0$, is the Poisson weight: $\sum_{x=0}^{\infty} p_{k}(x) p_{m}(x) j(x)=\delta_{k m}$.

Let $q(x)$ be a real almost periodic function (in the Bohr sense) on $X$. Then the matrix

$$
M_{n}(q)=\left\{\sum_{x=0}^{\infty} p_{k}(x) p_{m}(x) q(x) j(x) ; k, m=0,1, \ldots, n-1\right\}
$$

is called the Toeplitz matrix, generated by the polynomials $\left\{p_{m}\right\}$ and the function $q$.

Here we prove the following conjecture of Grenander and Szegö [2, p. 174]:

$$
\lim _{n \rightarrow+\infty} \frac{1}{n} \operatorname{trace}\left[M_{n}(q)\right]^{k}=\lim _{n \rightarrow+\infty} \frac{1}{n} \sum_{x=0}^{n-1}[q(x)]^{k}, \quad k=0,1, \ldots
$$

As a consequence one obtains the asymptotic distribution of the spectrum of the matrix $M_{n}(q)$ as $n \rightarrow+\infty$. Namely, let $N(\alpha, \beta, n)$ be the number of all eigenvalues of $M_{n}(q)$ lying on the segment $[\alpha, \beta]$. Define the distribution function $D(\alpha)$ by the lower limit: $D(\alpha)=\underline{\lim }_{n \rightarrow+\infty} \frac{1}{n} \sum_{x=0}^{n-1} \chi(\alpha-q(x))$ where $\chi$ is the characteristic function of the interval $(0, \infty)$. Then we have

$$
\lim _{n \rightarrow+\infty} \frac{1}{n} N(\alpha, \beta, n)=D(\beta)-D(\alpha)
$$

if $\alpha$ and $\beta$ are points of continuity for $D$.

Proof of (1). Let $E_{n}$ be the operator in $l^{2}$ with kernel

$$
e(n, x, y)=\sum_{m=0}^{n-1} p_{m}(x) p_{m}(y)
$$

Received by the editors June 20, 1990 .

1980 Mathematics Subject Classification (1985 Revision). Primary 47B35, 47B37; Secondary 47B10, 39A12. 
and let $Q$ be the operator of multiplication by $q$. Then we have

$$
\text { trace }\left[M_{n}(q)\right]^{k}=\text { trace } Q_{n}^{k}
$$

where $Q_{n}=E_{n} Q E_{n}$. Indeed, if $T$ is the infinite matrix $M_{\infty}(q)$, considered as an operator in $l^{2}$, then it is not hard to see that

$$
\text { trace }\left[M_{n}(q)\right]^{k}=\operatorname{trace} T_{n}^{k}
$$

where $T_{n}=P_{n} T P_{n}$ and $P_{n}$ is the operator of multiplication by the characteristic function of the set $\{0,1, \ldots, n-1\}$. On the other hand, the operators $T_{n}$ and $Q_{n}$ are unitary equivalent: $Q_{n}=F^{-1} T_{n} F$ where $F$ is the Fourier transform with respect to the complete system $\left\{p_{m}\right\}$, that is, $(F u)(m)=$ $\sum_{x=0}^{\infty} p_{m}(x) u(x) j(x)$. Thus (4) follows from (5).

Further, the operator $Q_{n}$ has a kernel

$$
Q_{n}(x, y)=\sum_{z=0}^{\infty} e(n, x, z) e(n, z, y) q(z) j(z)
$$

and $E_{n}$ is an orthogonal projection. Therefore

$$
\text { trace } Q_{n}=\sum_{x=0}^{\infty} e(n, x, x) q(x) j(x) .
$$

Since $Q_{n}^{2}=E_{n} Q^{2} E_{n}-S_{n}^{*} S_{n}$ where $S_{n}=\left(\mathrm{id}-E_{n}\right) Q E_{n}$, it follows that

$$
\operatorname{trace} Q_{n}^{2}=\sum_{x=0}^{\infty} e(n, x, x) q^{2}(x) j(x)-\left\|S_{n}\right\|_{2}^{2}
$$

where $\|\cdot\|_{2}$ stands for the Hilbert-Schmidt norm [1]. Analogously, $Q_{n}^{k}=$ $E_{n} Q^{k} E_{n}+S_{k, n}$ where $S_{k, n}$ is a sum of $2^{k-1}-1$ terms, each of them containing as a cofactor $S_{n}^{*}$ and $S_{n}$. Therefore

$$
\begin{gathered}
\operatorname{trace} Q_{n}^{k}=\sum_{x=0}^{\infty} e(n, x, x) q^{k}(x) j(x)+R_{n, k}, \quad k \geq 1, \\
\left|R_{n, k}\right| \leq\left(2^{k-1}-1\right)\|q\|^{k-2}\left\|S_{n}\right\|_{2}^{2}, \quad R_{n, 1}=0
\end{gathered}
$$

where $\|q\|=\sup _{x \in X}|q(x)|$. Further, the operator $S_{n}$ has a kernel

$$
S_{n}(x, y)=\sum_{z=0}^{\infty}(q(x)-q(z)) e(n, x, z) e(n, z, y) j(z),
$$

hence

$$
\left\|S_{n}\right\|_{2}^{2}=\frac{1}{2} \sum_{x=0}^{\infty} \sum_{y=0}^{\infty}(q(x)-q(y))^{2}|e(n, x, y)|^{2} j(x) j(y) .
$$

Thus, the formulas (6)-(8) show that it suffices to know the asymptotics of the function $e(n, x, x)$ as $n \rightarrow+\infty$ and an estimate of $e(n, x, y)$ if $x \neq y$ and $n \rightarrow+\infty$. We shall prove the following uniform asymptotics and estimates, which are sufficient for our purposes:

Case $1.0 \leq x \leq n\left(1-n^{-\delta}\right)$, where $0<\delta<1 / 4$.

$$
e(n, x, x) j(x)=1+e^{-\sqrt{n}} O(1), \quad n \rightarrow+\infty ;
$$


Case 2: $x \geq n\left(1+n^{-\delta}\right)$.

$$
e(n, x, x) j(x)=n^{\delta} \exp \left(\frac{n}{1+n^{\delta}}-\frac{x n^{\delta}}{\left(1+n^{\delta}\right)^{2}}\right), \quad n \rightarrow+\infty ;
$$

Case 3. $0 \leq y<x \leq n\left(1-n^{-\delta}\right)$.

$$
((x-y) e(n, x, y))^{2} j(x) j(y)=n e^{-\sqrt{n}} O(1), \quad n \rightarrow+\infty ;
$$

Case 4. For all $x$ and $n$

$$
e(n, x, x) j(x)<1 \text {. }
$$

Before proving these properties, we shall show that (4) and (6)-(12) imply (1). Namely, it is not hard to see that

$$
\sum_{x=0}^{\infty} e(n, x, x) q^{k}(x) j(x)=\sum_{x=0}^{n-1} q^{k}(x)+\|q\|^{k} o(n), \quad n \rightarrow+\infty .
$$

On the other hand,

$$
\begin{aligned}
\left\|S_{n}\right\|_{2}^{2} \leq & \sum_{x=1}^{n\left(1-n^{-\delta}\right)} \sum_{y=0}^{x-1}(q(x)-q(y))^{2}|e(n, x, y)|^{2} j(x) j(y) \\
& +4\|q\|^{2} \sum_{x=n\left(1-n^{-\delta}\right)}^{\infty} e(n, x, x) j(x) .
\end{aligned}
$$

Therefore, if $q$ is a Lipschitz function with a norm

$$
\|q\|_{1}=\sup _{x, y}|q(x)-q(y)||x-y|^{-1}+\|q\|,
$$

we obtain

$$
\left\|S_{n}\right\|_{2}^{2}=\|q\|_{1}^{2} o(n), \quad n \rightarrow+\infty .
$$

Thus (6)-(8), (13), and (14) yield the asymptotics

$$
\operatorname{trace} Q_{n}^{k}=\sum_{x=0}^{n-1} q^{k}(x)+2^{k}\|q\|^{k-2}\|q\|_{1}^{2} o(n), \quad n \rightarrow+\infty .
$$

Hence, (15) and (4) imply (1) if $q$ is a Lipschitz function. It remains to notice that the Lipschitz class is a dense set in the space of all almost periodic functions with respect to the supremum norm $\|q\|$. Thus (1) is proved.

Proof of (9)-(12). We shall use the formula

$$
e(\lambda, x, y)=\frac{1}{2 \pi i} \int_{\varepsilon-i \pi}^{\varepsilon+i \pi} e^{\lambda w} U(w, x, y) H(\lambda, w) d w, \quad \varepsilon>0,
$$

where $e(\lambda, x, y)$ is the step function: $e(\lambda, x, y)=e(n, x, y)$ if $n \leq \lambda<$ $n+1, n=1,2, \ldots$, and $e(\lambda, x, y)=0$ if $\lambda<1$. Here

$$
U(w, x, y)=\int_{0}^{\infty} e^{-\lambda w} d e(\lambda, x, y)=\sum_{n=0}^{\infty} e^{-w(n+1)} p_{n}(x) p_{n}(y)
$$


is an entire $2 i \pi$-periodic function with respect to $w$, and moreover (see [5]) for $y \geq x$ we have:

$$
U(w, x, y)=\exp \left(a e^{-w}\right) \sum_{k=0}^{x}\left(\begin{array}{l}
x \\
k
\end{array}\right)\left(\begin{array}{l}
y \\
k
\end{array}\right) k ! a^{-k} e^{-w k}\left(1-e^{-w}\right)^{x+y-2 k} .
$$

In particular, $\sum_{n=0}^{\infty} p_{n}^{2}(x)=U(0, x, x)=1 / j(x)$, whence the estimate (12) follows immediately. Finally, the function $s \rightarrow H(s, w)$ is 1-periodic and $H(s, w)=\frac{1}{2}\left(\operatorname{sh} \frac{w}{2}\right)^{-1} \exp \left(\frac{1}{2}-s\right) w$ if $0 \leq s<1$.

For proving (16), we use the relation

$$
w^{-1} U(w, x, y)=\int_{0}^{\infty} e^{-\lambda w} e(\lambda, x, y) d \lambda .
$$

Since the function $\lambda \rightarrow e(\lambda, x, y)$ is continuous only from the right, we pass to the average:

$$
e_{h}(\lambda, x, y)=\frac{1}{h} \int_{0}^{h} e(\lambda+\mu, x, y) d \mu, \quad h>0 .
$$

Notice that $e_{h}(\lambda, x, y) \rightarrow e(\lambda, x, y)$ as $h \rightarrow+0$ for every $(\lambda, x, y)$. From (17) it follows that

$$
\frac{e^{h w}-1}{h} \frac{U(w, x, y)}{w^{2}}=\int_{0}^{\infty} e^{-\lambda w} e_{h}(\lambda, x, y) d \lambda, \quad \operatorname{Re} w>0,
$$

so the inverse Laplace formula gives the equality

$$
e_{h}(\lambda, x, y)=\frac{1}{2 \pi i} \int_{\varepsilon-i \infty}^{\varepsilon+i \infty} e^{\lambda w} \frac{e^{h w}-1}{h} \frac{U(w, x, y)}{w^{2}} d w, \quad \varepsilon>0 .
$$

Taking into account the periodicity of the function $w \rightarrow U(w, x, y)$, we obtain

$$
e_{h}(\lambda, x, y)=\frac{1}{2 \pi i} \int_{\varepsilon-i \pi}^{\varepsilon+i \pi} e^{\lambda w} U(w, x, y) \frac{g(h, w)-g(0, w)}{h} d w
$$

where $g(s, w)=e^{w s} f(\lambda+s, w)$ and $f(s, w)=\sum_{k=-\infty}^{\infty}\left(e^{i 2 s k \pi} /(w+2 i k \pi)^{2}\right)$. It is clear that the function $s \rightarrow f(s, w)$ is 1-periodic and

$$
f(s, w)=\frac{1}{4}\left(\operatorname{sh} \frac{w}{2}\right)^{-1}\left(\operatorname{cth} \frac{w}{2}-1+2 s\right) \exp \left(\frac{1}{2}-s\right) w \quad \text { if } 0 \leq s<1 .
$$

Therefore, $\lim _{h \rightarrow 0} h^{-1}(g(h, w)-g(0, w))=H(\lambda, w)$, and the Lebesgue limit theorem is applicable. So (16) follows from (18).

Further, we have the estimate

$$
|j(x) U(w, x, x)| \leq \exp \left(4 \sqrt{a x}\left|\operatorname{sh} \frac{w}{2}\right|-x \operatorname{Re} w\right) \exp \left(a e^{-\operatorname{Re} w}-a\right) .
$$

Indeed, it is obvious that

$$
U(w, x, x)=\exp \left(a e^{-w}\right) a^{-x} x ! e^{-w x} \sum_{k=0}^{x}\left(\begin{array}{l}
x \\
k
\end{array}\right) \frac{1}{k !}\left(2 \sqrt{a} \operatorname{sh} \frac{w}{2}\right)^{2 k} .
$$

Since $\left(\begin{array}{l}x \\ k\end{array}\right) \leq x^{k}(k !)^{-1}$ and $\sum_{k=0}^{x}(k !)^{-2}\left(2 \sqrt{a x}\left|\operatorname{sh} \frac{w}{2}\right|\right)^{2 k} \leq \exp \left(4 \sqrt{a x}\left|\operatorname{sh} \frac{w}{2}\right|\right)$, the estimate (19) holds. 
To prove the asymptotic bound (10), we use formula (16) with $\varepsilon=\left(1+n^{\delta}\right)^{-1}$ where $0<\delta<1 / 4$. Since $x>n(1-\varepsilon)^{-1}$, we have $x>16 a \varepsilon^{-4} \operatorname{ch}^{2} \frac{\varepsilon}{2}$ if $n^{1-4 \delta}>\left(1+n^{-\delta}\right)^{3} 16 a \operatorname{ch}^{2} \frac{\varepsilon}{2}$, therefore (19) yields

$$
|j(x) U(w, x, x)| \leq e^{-\varepsilon(1-\varepsilon) x} \quad \text { if } x>n\left(1+n^{-\delta}\right), n>C(\delta, a) .
$$

Taking into account the bound $|H(n, w)| \leq C \varepsilon^{-1}$ if $\operatorname{Re} w=\varepsilon$, we obtain (10) from (16) and (20).

For proving (9), we notice that the function $w \rightarrow e^{\lambda w} U(w, x, y) H(\lambda, w)$ is $2 i \pi$-periodic, so the Cauchy formula and (16) imply

$$
e(n, x, x)=U(0, x, x)+\frac{1}{2 \pi i} \int_{-1-i \pi}^{-1+i \pi} e^{n w} U(w, x, x) H(n, w) d w .
$$

Since $x \leq n\left(1-n^{-\delta}\right), 0<\delta<1 / 4$, we have $4 \sqrt{a x}\left|\operatorname{sh} \frac{w}{2}\right|+x-n \leq-\sqrt{n}$ if $n>C(\delta, a), \operatorname{Re} w=-1$, so the estimate (19) with $\operatorname{Re} w=-1$ shows that

$$
|j(x) U(w, x, x)| \leq \exp (a e-a) e^{-\sqrt{n}} \text { if } x \leq n\left(1-n^{-\delta}\right) .
$$

Thus (9) follows from (21), since $j(x) U(0, x, x)=1$.

The proof of the estimate (11) is based on the Christoffel-Darboux formula:

$$
(x-y) e(n, x, y)=\sqrt{a n}\left(p_{n}(x) p_{n-1}(y)-p_{n-1}(x) p_{n}(y)\right),
$$

which follows from the recurrence relation [5]:

$$
\lambda_{n} p_{n+1}(x)=(x-a-n) p_{n}(x)-\lambda_{n-1} p_{n-1}(x), \quad \lambda_{n-1}=\sqrt{a n} .
$$

According to (9) we have

$$
j(x) p_{n}^{2}(x)=e^{-\sqrt{n}} O(1), \quad n \rightarrow+\infty \text { if } 0 \leq x \leq n\left(1-n^{-\delta}\right), 0<\delta<\frac{1}{4} .
$$

Therefore the estimate (11) is a consequence of (22) and (23).

Proof of (2). It is sufficient to consider the points $\alpha, \beta$ on the segment $[m, M]$, where $m=\inf q(x), M=\sup q(x)$. For the sequence of distribution functions $D_{n}(\alpha)=\frac{1}{n} \sum_{x=0}^{n-1} \chi(\alpha-q(x))$ the inequalities $0 \leq D_{n}(\alpha) \leq 1$ hold, hence there exists a limit $D_{1}(\alpha)=\lim _{j \rightarrow \infty} D_{n_{j}}(\alpha)$. Then formula (1) can be written in the form:

$$
\lim _{n \rightarrow \infty} \frac{1}{n} \operatorname{trace}\left[M_{n}(q)\right]^{k}=\int_{m}^{M} \alpha^{k} d D_{1}(\alpha) .
$$

Therefore, we can apply the method of Grenander and Szegö [2, p. 129] and conclude that

$$
\lim _{n \rightarrow \infty} \frac{1}{n} N(\alpha, \beta, n)=D_{1}(\beta)-D_{1}(\alpha)
$$

if $\alpha$ and $\beta$ are points of continuity for $D_{1}(\alpha)$. Since the functions $D_{1}(\alpha)$ and $D(\alpha)$ coincide on the set of the points $\{\alpha\}$ of continuity [4], formula (2) follows from (24).

\section{REFERENCES}

1. I. Gohberg and M. Krein, Introduction to the theory of linear nonselfadjoint operators, Amer. Math. Soc. Transl. of Math. Monographs, vol. 18, Amer. Math. Soc., Providence, RI, 1969.

2. U. Grenander and G. Szegö, Toeplitz forms and their applications, Moscow, 1961. 
3. V. Guillemin, Some classical theorems in spectral theory revisited, Ann. of Math. Stud. 91 (1979), 219-259.

4. B. Levitan, Almost periodic functions, Moscow, 1953.

5. J. Meixner, Erzengende Funktionen der Charlierschen Polynome, Math. Z. 44 (1938), 531535.

6. G. Szegö, Orthogonal polynomials, Amer. Math. Soc. Colloq. Publ. vol. 23, Amer. Math. Soc., Providence, RI, 1959.

7. H. Widom, Eigenvalue distribution theorems for certain homogeneous spaces, J. Funct. Anal. 32 (1979), 139-147.

Institute of Mathematics, Bulgarian Academy of Sciences, 1113 Sofia, Bulgaria 